\title{
The effect of the intervention dance program on the level of functional fitness and postural stability in the elderly
}

\author{
Pavlína Vaculíková', Alena Skotáková', \\ Sylvie Kropáčová ${ }^{3}$, Roman Grmela²
}

\author{
'Department of Gymnastics and Combatives, Faculty of Sports Studies, Masaryk University, Brno, Czech \\ Republic \\ ${ }^{2}$ Department of Health Promotion, Faculty of Sports Studies, Masaryk University, Brno, Czech Republic \\ ${ }^{3}$ Applied neuroscience research group, CEITEC - Central European Institute of Technology, Masaryk University, \\ Brno, Czech Republic
}

\begin{abstract}
Objective: The major objective of this study was to investigate the influence of six months dance intervention program on the level of functional fitness and postural stability in the elderly.

Methods: The exercise program was carried out three times a week and included 60 dance exercise sessions. 60 elderly participated in the study (67.5 \pm 5.69 years), 30 in a dance intervention group (weight: $70.32 \pm 13.59 \mathrm{~kg} ; B M I 25.95 \pm 3.79 \mathrm{~kg} / \mathrm{m}^{2}$ ), 30 in a control group (weight: $76.93 \pm 15.45 \mathrm{~kg} ; \mathrm{BMI}$ $27.06 \pm 3.88 \mathrm{~kg} / \mathrm{m}^{2}$ ). Three subtests (6Min Walk Test, Chair Stand Test, 8 Foot up and Go Test) were used to determine the level of functional fitness from the Senior fitness test battery; the static posturography method assessed the level of balance abilities in one position.

Results: The most meaningful results to emerge from the data are that the intervention dance program led to a post-exercise increase in the level of agility, dynamic balance and reaction speed measured by 8 Foot up Go test in the intervention group. The results indicate a statistically significant difference value in the intervention group compared to the control group between post-test results $(p=0.01)$. Values of the other monitored parameters were not statistically significant. The Cohen coefficient $d$ reflects the low (8 Foot up and Go test, 6 Min Walk test) or medium value (Chair Stand test) of the intervention programis effectiveness.

Conclusions: Our study suggests, that intervention dance program could significantly improve the level of agility, dynamic balance and reaction speed.
\end{abstract}

Keywords: aging, dance, Senior fitness test, motor abilities, balance ability

\section{INTRODUCTION}

It has been proven by many studies (Kalvach, 2004; Eijkeren et al., 2008; Uhliřr, 2008) that any regular physical activity is an effective strategy for healthy age/aging. It is one of the most important instruments used to achieve the quality of life in the elderly. Regular physical activity in the elderly population helps to slow down the onset of involution changes. Active seniors can stay self-sufficient and independent longer. Physical activity is also crucial in increasing the resilience of the elderly to disease, preventing certain civilization diseases and reducing the risk events that can lead to death (Ellingson \& Conn, 2000; Svobodová, 2017).

Including dance into the offer of physical activities broadens the range of options where everyone can find an activity to match their needs. Dancing has the potential to be an attractive physical activity that can be adjusted to fit a target population's age, physical limitations, and culture (Skotáková, Vaculíková, Sklenaříková \& Grmela, 2017). In recent years many authors (Federici, Bellagamba \& Rocchi, 2005; Hwang \& Braun, 2015; Keogh, Kilding, Pidgeon, Ashley 
\& Gillis, 2009) agree that dance in many varieties and styles could be a suitable activity for the group of the elderly.

Some dance intervention programs have been proposed to improve the balance ability and reduce falls in aging (Ferrufino, Bril, Dietrich, Nonaka, \& Coubard, 2011; Hui, Chui, \& Woo, 2009). Other studies focus on increasing the level of endurance, coordination and joint mobility. Their findings confirm the improvement in observed parameters due to the dance intervention program (Cruz-Ferreira, Marmeleira, Formigo, Gomes \& Fernandes, 2015; Zhang, IshikawaTakata, Yamazaki, Morita \& Ohta, 2008). A positive, statistically significant effect of the dance programs on the strength of the lower limbs, which can be the limiting factor in falls, is also demonstrated in several studies (Granacher et al., 2012; Merom et al., 2016; Zhang et al., 2008).

If we count the benefits of the dance, we cannot ignore psychological and sociological influence as in the studies of Dosedlová (2012), West, Otte, Geher, Johnson \& Mohr (2004) and Winkelmann et al. (2004). The social relations - fellowship among the participants of dance exercise programs, play a significant role in the quality of their lives. It is about creating pleasant experiences, positive feelings while dancing and also the pleasure of movement that the seniors themselves consider to be very important.

The aim of the contribution si to investigate the effect of the dance intervention program on the level of functional fitness and postural stability in a group of elderly. This article is one of the outputs of the project "The effect of an intensive dance-exercise intervention on cognitive functions and cerebral plasticity changes in healthy seniors and seniors with mild cognitive impairment”.

In another forthcoming publication (another output of the same project) entitled "DanceMovement Activities for Healthy Seniors and Seniors with Mild Cognitive Impairment”) we monitor the impact of this dance-exercise intervention on the same parameters in seniors divided, based on MRI (magnetic resonance imaging) and psycho-tests results, into healthy seniors and seniors with Mild Cognitive Impairment.

\section{METHODS}

\section{Participants}

Our sample consisted of 60 older adults (44 women, 16 men) over the age of $60(67.5 \pm 5.69$ years). Elderly participants were recruited using the snowball technique from several sources. A cooperating organization which provided education for the elderly (U3A, Senior academy, ...) were addressed, recruitment was also carried out free advertising in community newsletters and key places (libraries or local authorities).

\section{Inclusion criteria}

Based on a structured interview all participants were non-smokers, without any serious diseases that would impede the implementation of dance and movement activities in the specified scope and duration. None of the participants was engaged in a regular sports activity that is more frequently practiced or more demanding than our intensive dance-exercise intervention.

All participants were asked to sign an informed consent form approved by the Ethics Committee of Masaryk University, Brno. The Ethic committee application number was 13/2015.

The participants were randomly divided in 1:1 ratio. Half of the group (24 women, 6 men) underwent a 6-month dance-exercise intervention; the second half (20 women, 10 men) was a control group. Participants were asked to maintain their usual daily regimen with the same amount of physical activity and normal daily lifestyle. The descriptive characteristics (age, BMI, weight, number of men and women) of the study sample are shown in Table 1. 
Tab. 1: Descriptive characteristics of the study sample

\begin{tabular}{lccccc}
\hline & \multicolumn{2}{c}{ Experimental group } & & \multicolumn{2}{c}{ Control group } \\
\cline { 2 - 3 } \cline { 5 - 6 } & $M$ & $S D$ & & $M$ & $S D$ \\
\hline Age & 68.24 & 5.00 & & 67.19 & 6.70 \\
BMI & 25.73 & 3.60 & & 27.20 & 3.90 \\
Weight (kg) & 70.02 & 13.70 & & 77.03 & 15.20 \\
& & & & & \\
& $N$ & $\%$ & & $N$ & $\%$ \\
Male & 6 & 20 & & 10 & 33.3 \\
Female & 24 & 80 & & 20 & 66.6 \\
\hline
\end{tabular}

Note: $M$ - mean, $S D$ - standard deviation, $N$ - number, \% - percentage, BMI - body mass index

\section{Methods}

Three tests from the Senior fitness test (SFT) battery determined the level of functional fitness and one test on the "FootWork Pro" stabilometric platform assessed the level of postural stability. All three controlled functional tests were performed in a gym, the static posturography test and body composition measurements (BMI characteristics) were carried out in a laboratory. Body composition measurements were found out using the direct segmental multi-frequency bioelectrical impedance analyzer (Inbody 230).

All participants participated in the testing two times (two identical experimental tests and measurements before and after the six months of the intervention program). Firstly, the anthropometric characteristics were found out, followed by static posturography and functional tests. All tests and measurements lasted about 40 minutes.

Senior Fitness Test (SFT) was created to evaluate the individual components of functional fitness in older adults. Subsequent tests assessing the level of motor abilities that can be improved due to dance intervention program and that are also closely related to performing daily tasks were chosen.

- 30 Chair stand test: assessing the level of the strength of lower limbs,

- 6 Minute Walk test: assessing the level of endurance,

- 8 Foot up and Go test: assessing the level of agility, dynamic balance and reaction speed (Rikli \& Jones, 2013).

Static posturography allows a precise assessment of the static balance level. Due to measurements on the "FootWork Pro" stabilometric platform, the precise results indicating the foot pressure layout and its changes in feet during the measurement and center of pressure (COP) movements can be assessed and visualized (Amcube, 2015). Lower numerical values indicate the minor deviation of the center of gravity. We measured the center of pressure trajectory (COP), the center of gravity deflection in forward (DF) and right-hand directions (DR). For assessing the level of balance ability we used one standardized subtest:

For assessing the level of balance ability, we can use one standardized subtest:

- "Broad posture": the standing position with legs width of the pelvis, arms along the body, eyes open - holding the position for $30 \mathrm{~s}$ 


\section{Intervention program}

The intervention program was conceived as “a world tour.” Dances like polka, Irish dance, African dance, Greek dance and tango were performed. More famous dances such as Polka and Irish dance have been taught first. Later, more demanding dances were included in terms of both motor and rhythm.

The exercise program was carried out three times a week and included 50 dance exercise sessions. Each session (60 minutes) contained parts of dance choreography, preparatory and general exercises focused on developing a good posture, a breathing and relaxation exercises. An experienced trainer and an assistant led the individual lesson.

After each dance-movement class, feedback from the participating seniors was obtained using a standard evaluation sheet for each lesson. The evaluation included the opinions of the participants on the musical accompaniment, on the dance activity and also on the point of view of their physical and mental state during the whole class. The evaluation sheet contained four closed descriptive characteristics with a scale from 1 "strongly disagree" to 6 "strongly agree." The part of the evaluation was also the opened sentence: "I would recommend..." which offered participants to write their comments and recommendations.

Compliance with the intervention was registered and calculated as a percentage of the sessions attended. Subjects whose attendance was lower than $60 \%$ were not included in the data analyses.

\section{The more detailed description of the unit:}

The introductory part lasting about 10 minutes consisted of warm-up (e.g., movements around the gym performed on rhyme of the music, gait in different positions - on toes, on heels, on inner or external parts of feet, on bent knees...), mobilization exercises and dynamic stretching of main muscles with tendency to become shortened.

In the main part (30 $\mathrm{min})$ steps of dance choreography were taught. Steps were gradually linked into the short sequence and repeated several times concerning seniors' individual needs. After completing the new part, the sequences taught in previous lessons were repeated, and the whole routine was formed and repeated several times. At the end of the main part, the mastered dances were replayed too. To raise the level of difficulty, we introduced new stimuli, such as the initial layout of participants. The final part was aimed at balance exercises (using balance equipment - fit balls, over balls, bosu, ladder), and on strengthening exercises (targeted, e.g., on muscles responsible for a good posture and muscles with the tendency on weakness). All workouts could be alternated with respect to individual particularities of each participant.

The final part included the stretching and short relaxation including respiratory exercises.

\section{Statistical analyses}

For analysis of obtained data, we used IBM SPSS Statistics 25 software. The data were evaluated using descriptive statistics: arithmetic mean and standard deviation. The data were not normally distributed; therefore Wilcoxon and Mann-Whitney U test were used. To identify the statistical significance of the differences between pre-test and post-test in both groups the Wilcoxon sign rank test was used. Mann-Whitney U test was applied to the significant results, to identify if the significance survives between groups comparison. Statistical significance was set at $p \leq 0.05$. The Cohen coefficient $d$, representing the effect size, was assessed to compare the effect of the intervention dance program on all monitored variables. 


\section{RESULTS}

Table 2 shows the descriptive statistics (mean, standard deviation) and the statistical significance values of individual Senior fitness tests and postural stability measured before and after the intervention dance program in the intervention and the control group.

Tab. 2: Descriptive statistics functional fitness and balance values in intervention and control groups

\begin{tabular}{|c|c|c|c|c|c|c|}
\hline & \multicolumn{3}{|c|}{ Intervention group } & \multicolumn{3}{|c|}{ Control group } \\
\hline & $\begin{array}{l}\text { Pre-test } \\
M \pm S D\end{array}$ & $\begin{array}{c}\text { Post-test } \\
M \pm S D\end{array}$ & $p$-value & $\begin{array}{l}\text { Pre-test } \\
M \pm S D\end{array}$ & $\begin{array}{c}\text { Post-test } \\
M \pm S D\end{array}$ & p-value \\
\hline \multicolumn{7}{|l|}{ SFT } \\
\hline 6 Min Walk (m) & $588.16 \pm 77.4$ & $622 \pm 63.79$ & $0.000^{*}$ & $574.5 \pm 78.28$ & $601.79 \pm 90.85$ & $0.01^{*}$ \\
\hline Chair Stand (number) & $16.40 \pm 3.73$ & $18.30 \pm 3.58$ & $0.03^{*}$ & $15.66 \pm 3.53$ & $16.14 \pm 4.31$ & 0.79 \\
\hline 8 Foot up Go (s) & $4.93 \pm 0.99$ & $4.64 \pm 0.80$ & $0.01^{*}$ & $5.06 \pm 0.90$ & $5.17 \pm 1.39$ & 0.82 \\
\hline \multicolumn{7}{|l|}{ LB } \\
\hline $\mathrm{COP}(\mathrm{cm} 2)$ & $2.65 \pm 1.47$ & $2.78 \pm 2.76$ & 0.26 & $2.08 \pm 1.47$ & $2.58 \pm 2.76$ & 0.42 \\
\hline $\mathrm{RD}(\mathrm{cm})$ & $1.54 \pm 0.72$ & $1.39 \pm 0.58$ & 0.22 & $1.25 \pm 0.54$ & $1.48 \pm 0.86$ & 0.31 \\
\hline $\mathrm{FD}(\mathrm{cm})$ & $2.23 \pm 0.99$ & $2.16 \pm 1.09$ & 0.20 & $1.98 \pm 0.65$ & $2.01 \pm 1.02$ & 0.76 \\
\hline
\end{tabular}

Note: $M$ - Mean, $S D$ - standard deviation, SFT - Senior fitness test, $L B$ - level of balance, COP - Center of pressure, $R D$ - Center of gravity, deflection in the right-hand direction, FD - Center of gravity deflection in the forward direction, $p$-value - statistical significance, ${ }^{*} p \leq 0.05$

The table 2 shows that both groups increased significantly the level of aerobic endurance measured by 6 Min Walk test in comparison with the pre-test (experimental group $p=0.000$, control group $\mathrm{p}=0.001$ ).

The tendency to improve we can also observe in the Chair Stand test assessing the level of strength of lower limbs and in the level of agility, reaction speed and dynamic balance measured by 8 Foot-Up-and-go Test. The intervention group increased the level of these abilities significantly ( $p=0.03$ in Chair stand test, $p=0.01$ in 8 Foot-Up-and-go Test). In the control group there is the only slight, non significant, enhancement $(p=0.79$ in Chair stand test, $p=0.82$ in 8 FootUp-and-go Test).

Regarding postural stability there were no statistically significant changes in post-test compared with pre-test in either group after 6 months.

\section{The effectiveness of the intervention dance program: SFT and the level of balance}

For greater clarity, the obtained results of the level of functional fitness and postural stability will be interpreted separately.

Table 3 presents the results of the effectiveness of the intervention dance program in Senior fitness tests and balance level indicated by p-value (statistical significance) and Cohen coefficient $d$ (Effect size). 
Tab. 3: The effectiveness of the intervention dance program: SFT and the level of balance

\begin{tabular}{|c|c|c|c|c|c|}
\hline & & \multicolumn{2}{|c|}{ p-value } & \multicolumn{2}{|c|}{ Cohen's d } \\
\hline & & $\begin{array}{l}\text { experimental } \\
\text { group }\end{array}$ & $\begin{array}{l}\text { control } \\
\text { group }\end{array}$ & $\begin{array}{l}\text { experimental } \\
\text { group }\end{array}$ & $\begin{array}{l}\text { control } \\
\text { group }\end{array}$ \\
\hline \multicolumn{6}{|c|}{ SFT } \\
\hline & 6 Min Walk (m) & 0.28 & & 0.2 & \\
\hline & Chair Stand (number) & 0.21 & & 0.5 & \\
\hline & 8 Foot up Go (s) & $0.01 *$ & & 0.4 & \\
\hline \multicolumn{6}{|c|}{ LB } \\
\hline & $\operatorname{COP}\left(\mathrm{cm}^{2}\right)$ & 0.18 & & 0.0 & \\
\hline & $\mathrm{RD}(\mathrm{cm})$ & 0.23 & & 0.1 & \\
\hline & $\mathrm{FD}(\mathrm{cm})$ & 0.30 & & 0.1 & \\
\hline
\end{tabular}

\section{Functional fitness}

The results (Table 3) show that the intervention dance program affected the most the level of agility, reaction speed and dynamic balance measured by 8 Foot-Up-and-go Test. In the intervention group, there was a significant change from the pre-test, and the result was also statistically significant compared to the change of the control group $(\boldsymbol{p}=\mathbf{0 . 0 1})$. The Cohen's $d$ value reaches 0.4 that reflects a low size effect.

Both groups increased the level of aerobic endurance measured by 6 Min Walk test in comparison with the pre-test, but the result is not statistically significant $(p=0.2)$. The value of Cohen's $d$ indicates a low size effect (0.2).

The same tendency we can observe in the Chair Stand test assessing the level of strength of lower limbs. Intervention group improved the level of this ability, but the result is not statistically significant compared to the control group ( $p=0.21)$. Cohen's coefficient $d$ value exceeds 0.5 that indicates the medium level of effect size.

\section{Postural stability}

Based on the results (Table 3) we can confirm there are no statistically significant changes in any monitored parameters (COP, RD, FD) in the "broad posture" test that finds out the level of static balance. The value of Cohen coefficient $d$ in all reliable (COP, RD, FD) indicates a low effect.

\section{DISCUSSION}

Recently, the quality of life in senior age is often discussed (Ellingson \& Conn, 2000, Von Bonsdorff, 2016). Due to the aging of the population, the necessity of regular physical activity in the elderly is often mentioned (Kalvach, 2004, Eijkeren et al., 2008). The appropriate physical activities can slow down the aging process and thus to contribute to increase or to maintain the good level of the quality of life.

In our research, the statistically significant values of 8 Foot up and Go test showed the increasing level of this monitored parameter in the intervention group compared to the control group ( $p=0.01$, Cohen's $d=0.4$ ). The research of Kattenstroth et al. (2010) suggests the same effect of the dance intervention program on the experimental group in comparison with the con- 
trol group. 8 Foot up and Go test evaluates the level of agility, dynamic balance and reaction speed. These abilities are used in day-to-day activities, in fast body position changes while, e.g., working in the kitchen or shopping. The increasing level of these abilities in our intervention group could be caused by frequent direction changes during choreography, by the necessity of space orientation to follow the choreography and also by using some balance demanding positions.

Although the resulting values of $\mathbf{6}$ Min Walk test did not reveal any statistically significant changes ( $p=0.28$, Cohen's $d=0.2$ ) in the intervention group compared to control group, we have to mention the growth of the level of endurance ability in the intervention group. This positive tendency may be due to the fact that the main part of the session lasted for 30 minutes and the steps, dance sequences or already mastered dances were replayed many times. The results of the control group could be influenced by the season in which the measurements took part. The post-test measurements were held in spring, when seniors are engaged in gardening or starting to walk in the countryside. The positive effect of the dance intervention programs on the endurance ability in seniors is mentioned in many types of research (Cruz-Ferreira et al., 2015; Hui et al., 2009). Similarly, Shanahan et al. (2016) in the results of their study suggest that the regular participation of seniors in dance sessions is associated not only with the increasing level of endurance ability but also by raising of balance ability and with many health benefits and also by improving the quality of life.

The result of the Chair stand test, assessing the level of lower limbs strength, did not show any statistically significant value ( $p=0.21$ ) compared to the control group. Cohen's coefficient $d$ value indicates the medium level of effect size (Cohen's $d=0.5$ ). Even though the dance intervention program was not specifically aimed at developing the level of lower limb strength, the experimental group was able to improve this parameter. The growing tendency could result from the use of dance steps and some exercises in the final part of the session on bent knees. Due to this fact, the level of lower limbs strength could increase - the similar results we can see in Kattenstroth at al. (2013) research. Six months of dance intervention program led to improving the level of this monitored parameter in the experimental group, unlike the control group that worsened in this test. The positive, statistically significant effect of the lower limb dance programs is also demonstrated in studies of Zhang et al. (2008) or Merom et al. (2016). The sufficient level of the strength ability is necessary e.g., climbing stairs, rising from the chair, getting out of the car and also plays a crucial role in fall prevention.

Concerning the level of static balance assessed by "broad posture" test, we did not find out any statistically significant differences any monitored parameters (COP, RD, FD). The level of effect size in all reliable (COP, RD, FD) indicates a low effect. Unlike our research, another study (Sofianidis et al. 2009) has shown an increasing level of balance ability due to the dance intervention program (the Greek dance). The positive effects of Caribbean dance on the level of static balance, joint mobility, strength and coordination is mentioned in the study of Federici, Bellagambam \& Rocchi (2005). According to Koegh et al. (2009) the main benefit of dance in the elderly is to improve the level of balance ability and reduce the risk of falls.

\section{The study limitations}

The pre-test results could be caused by the ignorence of the tests. In post-test measurements the learning effect could positively influence the results. Furthermore, the different level of motivation of the tested sample was observed. This fact could be affected by the current mental and physical state of mind. The results could also be influenced by the season in which the measurements took part. The post-test measurements held in spring, when seniors are engaged in gardening or starting to walk in the countryside. We can assume that their fitness level may be affected by this fact. 


\section{CONCLUSION}

Our study suggests, that intervention dance program significantly improved the level of agility, dynamic balance and reaction speed. The level of aerobic endurance, the same as the level of leg strength did not reveal any statistically significant enhancement. However, in both mentioned parameters (aerobic endurance and the lower limb strength) we could see a slight improvement. Based on the results there are no statistically significant changes in the level of postural stability. Even if the dance intervention program did not have a more significant effect on the level of all tested parameters, we must state that only a slight improvement or a steady level of functional fitness and postural stability is a positive result in this age.

Apart from the importance of maintaining the level of physical fitness in senior age at a sufficient level, the positive impact of dance on psychological and sociological parameters must not be neglected. It is precisely the effect of the dance program on the chosen psychological characteristics and their link with the change in the quality of life of seniors, or life satisfaction, could be another topic of the research work.

\section{Acknowledgments}

This article was written at Masaryk University in cooperation with the Central European Institute of Technology (CEITEC) and the support of the Ministry of Health of the Czech Republic as a part of the project nr. 15-33854A "The effect of an intensive dance-exercise intervention on cognitive functions and cerebral plasticity changes in healthy seniors and seniors with mild cognitive impairment.” All rights reserved.

\section{REFERENCES}

Cruz-Ferreira, A., Marmeleira, J., Formigo, A., Gomes, D., \& Fernandes, J. (2015). Creative Dance Improves Physical Fitness and Life Satisfaction in Older Women. Research on Aging, 37(8), 837-855. https://doi.org/10.1177/0164027514568103.

Dosedlová, J. (2012). Terapie tancem. Praha: Grada Publishing a. s.

Ellingson, T., \& Conn, V. S. (2000). Exercise and quality of life in elderly individuals. Journal of Gerontological Nursing, 26(3), 17-25.

Federici, A., Bellagamba, S., \& Rocchi, M. B. L. (2005). Does dance-based training improve balance in adult and young old subjects? A pilot randomized controlled trial. Aging Clinical and Experimental Research, 17(5), 385-389.

Ferrufino, L., Bril, B., Dietrich, G., Nonaka, T., \& Coubard, O. A. (2011). Practice of Contemporary Dance Promotes Stochastic Postural Control in Aging. Frontiers in Human Neuroscience, 5. https://doi.org/10.3389/fnhum.2011.00169.

Granacher, U., Muehlbauer, T., Bridenbaugh, S. A., Wolf, M., Roth, R., Gschwind, Y., ... Kressig, R. W. (2012). Effects of a Salsa Dance Training on Balance and Strength Performance in Older Adults. Gerontology, 58(4), 305-312. https://doi. org/10.1159/000334814.

Hui, E., Chui, B. T., \& Woo, J. (2009). Effects of dance on physical and psychological well-being in older persons. Archives of Gerontology and Geriatrics, 49(1), 45-50. https://doi.org/10.1016/j.archger.2008.08.006.

Hwang, P. W.-N., \& Braun, K. L. (2015). The Effectiveness of Dance Interventions to Improve Older Adults' Health: A Systematic Literature Review. Alternative therapies in health and medicine, 21(5), 64-70.

Kalvach, Z. (2004). Geriatrie a gerontologie. Praha: Grada Publishing, 2004. ISBN 80-247-0548-6.

Kattenstroth, J.-C., Kolankowska, I., Kalisch, T., \& Dinse, H. R. (2010). Superior Sensory, Motor, and Cognitive Performance in Elderly Individuals with Multi-Year Dancing Activities. Frontiers in Aging Neuroscience, 2 (31). https://doi.org/10.3389/ fnagi.2010.00031.

Kattenstroth, J.-C., Kalisch, T., Holt, S., Tegenthoff, M.\& Dinse, H. R. (2013). Six months of dance intervention enhances postural, sensorimotor, and cognitive performance in elderly without affecting cardio-respiratory functions. Frontiers in Aging Neuroscience, 2 (31). https://doi.org/10.3389/fnagi.2013.00005.

Keogh, J. W. L., Kilding, A., Pidgeon, P., Ashley, L., \& Gillis, D. (2009). Physical Benefits of Dancing for Healthy Older Adults: A Review. Journal of Aging and Physical Activity, 17(4), 479-500. https://doi.org/10.1123/japa.17.4.479.

Merom, D., Mathieu, E., Cerin, E., Morton, R. L., Simpson, J. M., Rissel, C., ... Cumming, R. G. (2016). Social Dancing and Incidence of Falls in Older Adults: A Cluster Randomised Controlled Trial. PLOS Medicine, 13(8). https://doi.org/10.1371/ journal.pmed.1002112.

Pávková, J. (2008). Pedagogika volného času: [teorie, praxe a perspektivy výchovy mimo vyučování a zařizení volného času. Praha: Portál. 
Rikli, R. E., \& Jones, C. J. (2013). Senior fitness test manual (2nd ed). Champaign, IL: Human Kinetics.

Shanahan, J., Coman, L., Ryan, F., Saunders, J., O'Sullivan, K., Ni Bhriain, O., \& Clifford, A. M. (2016). To dance or not to dance? A comparison of balance, physical fitness and quality of life in older Irish set dancers and age-matched controls. Public Health, 141, 56-62. https://doi.org/10.1016/j.puhe.2016.07.015.

Skotáková, A., Vaculíková, P., Sklenaříková, J., \& Grmela, R. (2017). The effect of dance interventions at the level of chosen physical abilities in elderly people. Review. In Sport and quality of life 11th International Conference on Kinanthropology, 29. 11.-1. 12. 2017. Brno: Masarykova univerzita.

Sofianidis, G., Dimitriou, A.-M., \& Hatzitaki, V. (2017). A Comparative Study of the Effects of Pilates and Latin Dance on Static and Dynamic Balance in Older Adults. Journal of Aging and Physical Activity, 25(3), 412-419.

Svobodova, L. (2017). Dance Contribution to Improving Physical Activity and Health. 8th International Scientific Conference on Kinesiology, 307-311.

Uhlî́, P. (2008). Pohybová cvičení seniorů. Olomouc: Univerzita Palackého v Olomouci.

Van Eijkeren, F. et al. (2008). Nordic walking improves mobility in Parkinson's disease. Movement disorders. Retrieved from: https://doi.org/10.1002/mds.22293.

Von Bonsdorff, M. B. (2016). Midlife work ability and mobility limitation in old age among non-disability and disability retirees. BMC Public Health, doi: 10.1186/s12889-016-2846-y.

West, J., Otte, C., Geher, K., Johnson, J., \& Mohr, D. C. (2004). Effects of Hatha yoga and African dance on perceived stress, affect, and salivary cortisol. Annals of Behavioral Medicine: A Publication of the Society of Behavioral Medicine, 28(2), 114-118. https://doi.org/10.1207/s15324796abm2802_6.

Winkelmann, A., Tharicharu, J., Lissner, P., Beauchet, O., Gold, G., \& Kressig, R. W. (2004). Exercise promotion among geriatric in-patients: Tai Chi and dance. Medecine Et Hygiene, 62(2484).

Zhang, J.-G., Ishikawa-Takata, K., Yamazaki, H., Morita, T., \& Ohta, T. (2008). Postural stability and physical performance in social dancers. Gait \& Posture, 27(4), 697-701. https://doi.org/10.1016/j.gaitpost.2007.09.004.

Amcube. (2015). Footwork Pro Pressure Plate, Pressure Measurement. Retrieved from 18. ŕíjen 2018, z http://www.amcube. co.uk/products/footwork-pro-pressure-plate/.

\section{Corresponding author:}

Mr. Pavlína Vaculíková, Ph.D

e-mail:vaculikova@fsps.muni.cz

tel: +420 777044419 